AperTO - Archivio Istituzionale Open Access dell'Università di Torino

\title{
A single cystatin C determination before coronary angiography can predict short and long-term adverse events
}

\section{This is the author's manuscript}

Original Citation:

Availability:

This version is available http://hdl.handle.net/2318/1731673

since 2020-02-27T12:31:33Z

Published version:

DOI:10.1016/j.ijcard.2019.09.069

Terms of use:

Open Access

Anyone can freely access the full text of works made available as "Open Access". Works made available under a Creative Commons license can be used according to the terms and conditions of said license. Use of all other works requires consent of the right holder (author or publisher) if not exempted from copyright protection by the applicable law. 
A single Cystatin $\mathrm{C}$ determination before coronary angiography can predict short and long-term adverse events

Carlo Budano ${ }^{\mathrm{a}}$,M.D.; Alessandro Andreis ${ }^{\mathrm{a}}$, M.D.; Ovidio De Filippo ${ }^{\mathrm{a}}$, M.D.; Arianna Bissolino $^{\text {a }}$ M.D.; Giacomo Lanfranco ${ }^{d}$, M.D.; Tullio Usmiania ${ }^{\mathrm{a}}$ M.D.; Massimo Gaid, M.D.;

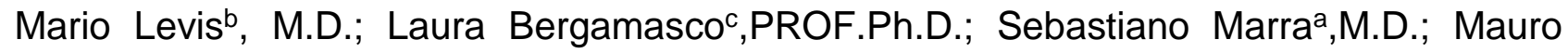
Rinaldia ${ }^{\mathrm{a}}$,PROF.M.D; Gaetano Maria De Ferraria ${ }^{\mathrm{a}}$,PROF.M.D;

(a) Division of Cardiology, A.O.U. Città della Salute e della Scienza di Torino-Molinette, Turin, Italy

(b) Department of Oncology, Radiation Oncology, University of Turin, Turin, Italy

(c) Department of Surgical Sciences, University of Turin, Turin, Italy

(d) Nephrology Department, A.O.U. Città della Salute e della Scienza di Torino, Turin, Italy

Carlo Budano: This author takes responsibility for all aspects of the reliability and freedom from bias of the data presented and their discussed interpretation.

Alessandro Andreis: This author takes responsibility for all aspects of the reliability and freedom from bias of the data presented and their discussed interpretation.

Ovidio De Filippo: This author takes responsibility for all aspects of the reliability and freedom from bias of the data presented and their discussed interpretation.

Arianna Bissolino: This author takes responsibility for all aspects of the reliability and freedom from bias of the data presented and their discussed interpretation.

Giacomo Lanfranco: This author takes responsibility for all aspects of the reliability and freedom from bias of the data presented and their discussed interpretation. 
Tullio Usmiani: This author takes responsibility for all aspects of the reliability and freedom from bias of the data presented and their discussed interpretation.

Massimo Gai: This author takes responsibility for all aspects of the reliability and freedom from bias of the data presented and their discussed interpretation.

Mario Levis: This author takes responsibility for all aspects of the reliability and freedom from bias of the data presented and their discussed interpretation.

Laura Bergamasco: This author takes responsibility for all aspects of the reliability and freedom from bias of the data presented and their discussed interpretation.

Sebastiano Marra: This author takes responsibility for all aspects of the reliability and freedom from bias of the data presented and their discussed interpretation.

Mauro Rinaldi: This author takes responsibility for all aspects of the reliability and freedom from bias of the data presented and their discussed interpretation.

Gaetano Maria De Ferrari: This author takes responsibility for all aspects of the reliability and freedom from bias of the data presented and their discussed interpretation.

Conflicts of interest: none declared

Keywords: Cystatin C; contrast induced acute kidney injury (CIAKI); contrast media; coronary angiography

Corresponding author:

Alessandro Andreis, MD

Division of Cardiology

AOU Città della Salute e della Scienza di Torino, Italy

Corso Bramante 88, 10126 Turin, Italy

Phone: +390116335945; +390116335945. 
e-mail: alessandro.andreis@unito.it

ABSTRACT (250 words)

Background. Cystatin C (CyC) role in the detection of contrast induced acute kidney injury $(\mathrm{CIAKI})$ is controversial. This study assessed whether a single CyC determination before coronary angiography (CA)could predict CIAKI and long-term adverse events.

Methods CyC was assessed before CA in 713 consecutive patients. CIAKI was the primary endpoint, defined as $\geq 0.3 \mathrm{mg} / \mathrm{dl}$ creatinine ( $\mathrm{SCR}$ ) increase at $48 \mathrm{~h}$ or $\geq 50 \%$ in 7 -days. Allcause death,cardiovascular (CV)death and MACE (acute coronary syndrome,acute pulmonary edema,CV death) were secondary endpoints. Re-hospitalization, in-hospital death and worsening renal function were tertiary endpoints.

Results CIAKI occurred in 47(6.7\%) patients. ROC analysis showed a good accuracy of CyC in the prediction of CIAKI (AUC 0.82,p<0.01), compared with baseline sCR and sCR-eGFR (AUC 0.70 and 0.75 respectively, both $\mathrm{p}<0.01$ ). CyC was associated with 10 -year CV-death, 
all-cause death and MACEs (AUC 0.76,0.74 and 0.64 respectively, all $p<0.01$ ). A CyC cutoff value of $1.4 \mathrm{mg} / \mathrm{L}$ was not only accurate in predicting or ruling-out CIAKI following CA ( $97 \%$ negative predictive value, $84 \%$ specificity), but also useful as a prognostic marker for 10-year adverse events (50\% vs.16\% all cause mortality, $29 \%$ vs.3\% CV death, $39 \%$ vs.13\% MACE,all $p<0.01)$, re-hospitalizations (54\% vs.35\%,p<0.01) and worsening renal function (34\% vs.19\%,p<0.01).

The strongest and independent risk factor for 10-year CV death was baseline CyC>1.4 mg/L (HR 17.3, 95\% Cl 1.94-155.1).

Conclusions A baseline determination of CyC before CA can accurately rule out CIAKI and predict adverse events in the long term. CIAKI can be ruled out before CA in $97 \%$ patients with a CyC value $<1.4 \mathrm{mg} / \mathrm{L}$. 


\section{INTRODUCTION}

Chronic kidney disease (CKD) is an independent risk factor for coronary artery disease(1). Ageing is physiologically associated with a renal function decline $(8 \mathrm{~mL} / \mathrm{min} / 1.73$ m2 estimated glomerular filtration rate (eGFR) reduction/decade after 30 years of age (2)). Coronary angiography $(\mathrm{CA})$ or percutaneous intervention $(\mathrm{PCl})$ is challenging in this setting, because of an increased risk of contrast-induced acute kidney injury (CIAKI)(3-5), defined as a serum creatinine $(\mathrm{sCr})$ increase of $\geq 0.3 \mathrm{mg} / \mathrm{dl}$ within 48 hours or $\geq 50 \%$ within 7 days following procedure (6). Such iatrogenic complication is the third cause of hospital-acquired acute renal failure $(7,8)$, worsening the prognosis $(3,5,9)$. Serum creatinine is widely used as a marker of renal function, even more accurate when computed in one of the apposite algorithms to estimate eGFR. However, sCr has a slow distribution kinetics among different body compartments, thus up to 72 hours-several days may be required to observe its rise in case of kidney damage; moreover, its blood levels are influenced by body mass, dietary intake, aging and they may not vary until about $50 \%$ of renal function has been lost (10). Therefore, SCR has some pitfalls in the identification of acute changes in renal function. Over the past decades the discovery and validation of biomarkers of kidney injury has gained significant interest. Cystatin $\mathrm{C}(\mathrm{CyC})$ is a protease inhibitor synthesized and released into the blood by all nucleated cells, freely filtered by kidney glomerulus and almost completely reabsorbed and metabolized by proximal tubule, not secreted. It is not affected by age, gender, race or muscle mass. Thanks to these properties, very small changes in glomerular filtration rate (GFR) may significantly influence CyC serum levels, potentially making this protein a very sensitive marker of renal filtration (11).

Studies comparing CyC with SCR in the early diagnosis of kidney injury after CM administration obtained controversial results (12-16). The vast majority of such studies assessed only CyC diagnostic value to identify CIAKI, but did not evaluate CyC prognostic role. A prospective study by Briguori et al. (13) on 410 stage 3-4 CKD patients undergoing 
CA showed CyC early variations after CM exposure were not only a good marker for the early diagnosis of CIAKI but also a reliable predictor of 1-year prognosis.

The aims of this study were to assess whether a single baseline CyC determination before CA could predict: 1) CIAKI occurrence following CA 2) long-term adverse events.

\section{METHODS}

Study design

All patients admitted to our institution between February 2006 and February 2007, at least 18 years old, with clinical indication to CA were included in the present prospective cohort study. Cardiogenic shock on admission, mechanical ventilation, simultaneous cancer disease and multiple myeloma were the only exclusion criteria.

Procedural management

Decisions concerning timing and characteristics of CA procedure, including CM doses and arterial access (femoral or radial artery), were left to discretion of the operators. Iso-osmolar dimeric CM iodixanol (Visipaque, Amersham Health, Oslo, Norway) was used in all cases. Patients with a baseline eGFR $<60 \mathrm{ml} / \mathrm{min} / 1.73 \mathrm{~m}^{2}$ (computed according to the Chronic Kidney Disease EPIdemiology collaboration equation, 17) were given CIAKI prophylaxis according to our department protocol: in vein (i.v.) hydration with $\mathrm{NaCl} 0.9 \%$ isotonic saline solution (IS), infusion rate $1 \mathrm{ml} / \mathrm{kg}$ body weight per hour (reduced to $0.5 \mathrm{ml} / \mathrm{kg} / \mathrm{h}$ if LVEF<40\%) for 12 hours before CA and for 18-24 hours later. They were additionally given: $1.4 \%$ sodium bicarbonate solution (SB), infusion rate $3 \mathrm{ml} / \mathrm{kg} / \mathrm{h}$ for 1 hour before procedure and then $1 \mathrm{ml} / \mathrm{kg} / \mathrm{h}$ (maximum $110 \mathrm{ml} / \mathrm{h}$ ) for 6 hours later; oral $\mathrm{N}$-acetylcysteine (NAC) 1200 mg on procedure day and $1200 \mathrm{mg}$ the following day; oral Vitamin C $5000 \mathrm{mg}$ on procedure day and $5000 \mathrm{mg}$ the following day.

Laboratory tests were assessed at baseline and 24 to 72 hours after angiography. All patients not requiring more than 3 days of hospital stay were discharged with indication to 
sCr evaluation at 7 days in our laboratory. The conventional Jaffe alkaline picrate method was used for sCr measurement, with Beckman CX7 assay (GMI, Ramsey, Minnesota, USA). Serum CyC was measured 24 hours before CA by immunonephelometry with Dade Behring Cystatin C assay (Dade Behring Diagnostics, Marburg, Germany).

Anamnestic and clinical data about the index hospitalization and the follow-up were collected by patient interrogation, medical outpatient visits or medical records analysis. Informations on patients who were not contactable were obtained from electronic medical records or direct contact with referring primary care physician.

End-points

The primary endpoint was CIAKI occurrence, defined according to KDIGO guidelines (6), as a $\geq 0.3 \mathrm{mg} / \mathrm{dl}$ increase in $48 \mathrm{~h}$ or $\geq 50 \%$ in 7 days following CA. Secondary end-points were: all-cause death, cardio-vascular (CV) death, a combined end-point of major adverse cardiovascular events (MACE, defined as: acute coronary syndrome [ACS], acute pulmonary edema [APE], CV death). Re-hospitalization, in-hospital death and worsening renal function were evaluated as tertiary endpoints. The occurrence of worsening renal function was defined as: CKD class worsening, patients started on dialysis, kidney transplantation.

Statistical analysis

Continuous variables, presented as means and standard deviations, were compared by non-parametric tests: Mann-Whitney's test was used for independent data and Wilcoxon's signed-rank test for paired data (pre-post evaluations). Categorical variables, presented as counts and percentages, were compared using the chi-square test with Yates' correction or Fisher's exact test: the odds ratio (OR) was computed with its 95\% confidence interval $(\mathrm{Cl})$. All analyses were performed using the SPSS for Windows version 18.0 (SPSS, Inc., Chicago, Illinois) and a two-sided significance level of $<0.05$ was considered statistically 
significant. The survival probability and the freedom from adverse events were evaluated with the Kaplan-Meier curves, compared by the Mantel-Cox test. Univariate logistic analysis was used to determine the association between risk factors and CIAKI. Multivariate logistic regression and Cox regression analysis were performed to examine the effects of different possible confounding variables. The discriminant ability of a test was evaluated by the Receiver Operating Characteristics (ROC) curve analysis, a plot of true positive rates (sensitivity) versus false positive rates (1-specificity). The Area Under the Curve (AUC) measured the quality of discrimination, from 0.5 (poor) to 1 (excellent). The cut-off value to determine the presence/absence of a disease was obtained through the simultaneous maximization of sensitivity and specificity via their harmonic mean.

\section{RESULTS}

Seven hundred thirteen consecutive patients were enrolled in the present study. Preprocedural $\mathrm{CyC}, \mathrm{sCr}$ and post-procedural sCr were assessed in all patients. Baseline risk factors, characteristics, clinical indication to CA and renal function along with CIAKI incidence are shown in Table $1 \mathrm{~A}$ and $1 \mathrm{~B}$. Mean age was $66 \pm 11$, with a remarkable prevalence of cardiovascular risk factors. In particular, sub-population of patients with CyC baseline value $\geq 1.4 \mathrm{mg} / \mathrm{L}$ was significantly older ( $71 \pm 9$ vs $65 \pm 11$ years old, $p<0.001$ ) and showed a higher prevalence of hypertension (85.1 vs $75.3 \%, p<0.0001)$, diabetes $(17.2 \%$ vs $7.9 \%, p=0.004)$, dyslipidemia $(46.5 \%$ vs $32.9 \%, p<0.001)$ as compared to patients with baseline СyС $<1.4 \mathrm{mg} / \mathrm{L}$. In addition, the formers were more likely to have a reduced left ventricular ejection fraction $(22.4 \%$ vs. $8.8 \%, p<0.001)$ and an impaired renal function (eGFR < $60 \mathrm{ml} / \mathrm{min} / 1.73 \mathrm{~m} 2: 67.2 \%$ vs 8.6\%, p<0.0001). Patients with higher levels of baseline $\mathrm{CyC}$ also had a worse baseline renal function evaluated according to ordinarily used indexes as serum creatinine $(1.88 \pm 1.94 \mathrm{mg} / \mathrm{dl}$ vs $0.91 \pm 0.19 \mathrm{mg} / \mathrm{dL}, \mathrm{p}<0.001)$ and sCreGFR (51.6 \pm 23.9 vs. $82.7 \pm 19.9, p<0.001)$. Contrast nephropathy risk score was 
significantly higher in this sub-population as well ( $7 \pm 3.59$ vs $3.55 \pm 2.8, p<0.001)$. Volume of contrast media used during the index procedure was comparable among the two groups $(230 \pm 150 \mathrm{ml}$ vs $227.8 \pm 161.6 \mathrm{ml}, \mathrm{p}=0.88)$. CIAKI incidence was $47 / 713(6.7 \%)$. Over a 10year follow-up, all-cause death occurred in 23\% patients, CV death in 9\%. MACE occurred in $18 \%$ patients.

Baseline CyC as a predictor of CIAKI

ROC analysis showed a statistically significant correlation between CyC and CIAKI incidence (Figure 1, panel a). Figure 1 - panel b displays a comparison between baseline CyC and other markers of renal function evaluated before CM exposure as potential predictors of CIAKI (i.e. serum Creatinine and CyC or sCr-eGFR estimated according several equations). All the markers showed a statistical significance in predicting $\mathrm{CIAKI}$ $(p<0.001)$, but they differed for their AUC. CyC had an AUC of 0.82 whereas sCR-eGFR according to MDRD, CyC-eGFR and SCR had an AUC of $0.75,0.83$ and 0.70 respectively. A baseline value of serum $\mathrm{CyC} \geq 1.4 \mathrm{mg} / \mathrm{L}$ was found to be the best cut-off to predict poor long-term outcomes. With regard to CIAKI incidence, 30 patients (22\%) with a baseline $\mathrm{CyC} \geq 1.4 \mathrm{mg} / \mathrm{L}$ developed CIAKI, whereas only 17 (2.9\%) with $\mathrm{CyC}<1.4 \mathrm{mg} / \mathrm{L}$ experienced such complication (RR 7.62 [4.3-13.4], $\mathrm{p}<0.001$ ). This cut-off value proved the best specificity (84\%) and a high negative predictive value (97\%) to rule-out CIAKI as compared to standard markers of renal function. Optimal cut-off values were estimated for all other indexes of renal functionality previously examined by ROC analysis. Their sensitivity, specificity, PPV, NPV, LR+, LR- are reported in Table 2, compared with the CyC cut off of $1.4 \mathrm{mg} / \mathrm{L}$. The confidence and reliability of this cut-off was strictly related to baseline renal functionality. In particular, CIAKI incidence among patients with $\mathrm{CyC} \geq 1.4 \mathrm{mg} / \mathrm{L}$ and stage 1 CKD was 9\%, becoming $18 \%$ in patients suffering from a stage 2 CKD, $24.6 \%$ in those with a stage $3 a / b$ CKD, and $31.2 \%$ in patients with a $4 / 5$ CKD. 
CyC as predictor of poor long-term outcomes (all-cause death, CV death and MACE) Baseline CyC was found to be a quite accurate predictor of 10-year adverse events such as all-cause death, CV death and MACE (AUC 0.76, 0.74 and 0.64 respectively, all $p<0.01$ ). Patients with a baseline CyC $\geq 1.4 \mathrm{mg} / \mathrm{L}$ had worse outcomes over a 10-year follow-up, with a greater all-cause mortality (50\% vs. $16 \%, p<0.001)$, CV death $(29 \%$ vs. $3 \%, p<0.001)$, MACE (39\% vs. 13\%, p<0.001), as shown in Kaplan-Meier curves in Figure 2.

As to the prediction of 10-year CV death, the use of the above mentioned cut-off yielded a $59 \%$ sensitivity, $83 \%$ specificity, $98 \%$ negative predictive value.

A Cox regression analysis confirmed the independent predictive role of baseline CyC for CV death at follow-up (HR 17.3: 1.94-155.1, $\mathrm{p}=0.01$ ). Other variables showing an independent prognostic impact at multivariate analysis were CIAKI (HR 11.1: 4.05 - 30.5, $p<0.0001)$, LVEF<40\% (HR 4.22: 1.64-10.83, $p=0.003)$, diabetes (HR 3.36: $1.32-8.56, p=$ $0.01)$ and statin treatment (HR 0.24: $0.08-0.68, p=0.007)$, whereas CKD on dialysis, MEHRAN class $>2$, age $>75$ years, dyslipidemia and renal filtration impairment with GFR<60 $\mathrm{ml} / \mathrm{min}$ (evaluated according to several equations) resulted uninfluential (Table 3).

\section{Tertiary end-points}

The CyC cut-off value of $1.4 \mathrm{mg} / \mathrm{L}$ was tested to explore a possible association with tertiary endpoints such as re-hospitalizations in cardiology/nephrology departments, CKD worsening and in-hospital death (Table 4). A significant relationship of baseline CyC $>1.4$ $\mathrm{mg} / \mathrm{L}$ with re-hospitalizations (54\% vs 35\%, $\mathrm{p}<0.01$ ) and worsening renal function (34\% vs. $19 \%, \mathrm{p}<0.01)$. Although patients with baseline CyC $>1.4 \mathrm{mg} / \mathrm{L}$ had a non-significant trend towards CKD class worsening, they had a higher risk to start a dialysis treatment (9\% vs $0 \%, p<0.0001)$ and to undergo a kidney transplantation ( $3 \%$ vs $0 \%, p<0.01$ The relationship 
between CyC baseline value $<1.4 \mathrm{mg} / \mathrm{L}$ and in-hospital death was not significant (3\% vs $0.9 \%, p=0.07)$

\section{DISCUSSION}

Main findings of this study are:

a) Baseline CyC (before CA) can predict CIAKI more accurately than others renal functionality markers more used in the ordinary clinical practice, including serum Creatinine and serum creatinine based-eGFR.

b) CyC baseline value is an independent prognostic marker of poor long term outcomes such as all cause death, CV death and MACE. For this endpoints the best cutoff value was a baseline CyC $\geq 1,4 \mathrm{mg} / \mathrm{L}$.

c) CyC baseline value $\geq 1,4 \mathrm{mg} / \mathrm{L}$ proved to be most specific tool in predicting and ruling out CIAKI with a negative predictive value of $97 \%$. Moreover, this cut-off was reliable in identifying people with a higher risk of re-hospitalization and of worsening of CKD class.

To our knowledge the present research represents the first one to analyze the predictive value of a single baseline CyC on CIAKI and long term adverse outcomes in a large cohort of unselected patients over a long-time follow up period. The importance of searching reliable biomarkers that could confidently predict the risk of developing acute renal injury is unanimously shared because of the recognized impact of CIAKI on prognosis $(18,19)$ Since CyC serum levels raises more rapidly than $\mathrm{sCr}$ after $\mathrm{CM}$ exposure (11), multiple studies tried to evaluate the role of CyC variations, before and after CM exposure, in detecting or ruling out CIAKI , obtaining controversial results $(13-16,20)$. However only few studies, 
mostly limited by small sample sizes $(21,22)$, assessed instead the accuracy of a single CyC determination obtained before procedures requiring the use of iodinate contrast media in predicting renal injury. Results of the present study highlight the reliability of baseline CyC determination to identify patients with a higher risk of developing CIAKI. In accordance, a single-center Japanese study enrolling patients undergoing diagnostic $\mathrm{CA}$ or $\mathrm{PCl}$ showed that in patients with low baseline creatinine values (namely $\mathrm{SCR}<1.5 \mathrm{mg} / \mathrm{dL}$ ), CIN was not a negligible complication and that values of $\mathrm{CyC}$ above the upper reference limit of 1.04 mg/L were independently associated with CIN development (22). Despite the specificity of such cut-off was not addressed, this finding suggest a more sensitive power of CyC as compared to sCr in predicting contrast-mediated kidney injury. Similarly, a case-control study conducted among 60 patients performing $\mathrm{PCl}$ and designed to establish the best cutoff value of CyC for the early diagnosis of $\mathrm{AKI}$, found significant higher values of baseline CyC in patients experiencing such complication compared to controls, thus supporting our findings (23).

Our results disagree instead with findings of a previous study by Liu et al. (16) stating that a single determination of CyC had poor sensitivity and specificity in predicting CIAKI. The study by Liu et al. had anyway several limitations, as for example a small sample size (311 patients) and inclusion criteria that excluded patients with severe CKD, whereas our results suggest that CyC reliability is higher in people with a worse baseline renal function. An elegant research by Laders et al., based on a retrospective analysis of the Prospective Dialysis versus Diuresis trial, suggested that neither baseline Creatinine nor baseline CyC were by their own statistically related to CIAKI development, thus introducing the preinterventional CyC/Cr ratio as a new index to preannounce such complication (24). Despite this study respected the good purpose of identifying CIAKI patients at risk of CIAKI before $\mathrm{CM}$ exposure, it did not compared the new suggested index (CyC/Cr ratio) with eGFR computed according different equations, notoriously more accurate than creatinine by itself 
in defining renal function. Moreover, the intrinsic need of dosing two markers (CyC and Creatinine) to obtain their ratio is not apparently convenient.

Despite controversial results reported in literature, our data seems to unequivocally indicate the usefulness of a single CyC determination before CM exposure to predict or confidently rule out the risk of developing CIAKI. Moreover, by comparing ROC-plot AUC, CyC seems to be even more accurate than classically used markers as eGRF (computed according all most known algorithms) and serum Creatinine. In particular a baseline CyC cut-off of 1.4 $\mathrm{mg} / \mathrm{L}$ proved to be highly specific and able to rule out CIAKI with a reassuring negative predictive value.

Results of the present study also show that a CyC baseline determination was a powerful independent prognostic factor of CV death and MACE on a long-term follow up.

Kaplan-Meyer analysis showed a statistically significant higher rate of CV death within 8 years and MACE for people with a baseline value of $\mathrm{CyC} \geq 1,4 \mathrm{mg} / \mathrm{L}$. As for CIAKI, many studies evaluated the impact of CyC post-procedural increasing on long term prognosis $(13,25,26)$ whereas the validity of a single CyC measurement on long term outcome was not deeply explored by previous researches. An aged study by Ichimoto et al. first showed that in a small cohort of patients with ST-elevation myocardial infarction, elevated baseline CyC values were associated with more cardiovascular events, mainly re-hospitalizations for congestive heart failure (27). The mentioned study was however limited by a small sample size, a highly selected cohort of STEMI patients, and a short follow up (mean FU 5.6 months).

The most immediate hypothesis that could support our finding is obviously related to CyC as a measure of kidney function, so that it could be easily concluded that patients with a worse baseline renal functionality clearly have a worse prognosis. Our results (see tertiary endpoints) and other studies aforementioned, stating that CyC can assess the status of Kidney functionality better than $\mathrm{sCr}$ and eGFR, would support this simplistic but incisive 
conjecture. However, other interesting hypotheses can be suggested. Some studies indeed, found a relationship between CyC and C-reactive protein (28) and inducible myocardial ischemia (29). The Dallas Heart study showed instead that CyC is independently associated with left ventricular mass, concentricity and wall thickness (30). Moran et al. confirmed that Cystatin C levels are linearly associated with the incidence of systolic and diastolic HF and that this association persisted after adjustment for age, blood pressure, left ventricular hypertrophy, history of coronary heart disease, diabetes and other potential confounders (31). Results of meta-analysis including 9 studies and 38.854 patients strongly supported the independent association between elevated CyC serum levels and CV-mortality (32).In this context, it cannot be excluded that CyC could be an overall index of pathophysiological abnormalities accompanying kidney disease, beyond its role as a kidney function marker. According to this theory, CyC could be considered a useful marker to identify "high-risk patients" with a worse prognosis, thus requiring a careful observation. However, these theories should only be considered as hypothesis-generating in absence of specific and stronger evidences.

In conclusion, as minor endpoints of our study, we report a higher frequency of rehospitalizations in Cardiology/Nephrology department, a higher rate of in-hospital death and a worse prognosis of kidney disease in people with higher levels of baseline CyC. Even if these endpoints were not the primary aim of our research, they further stress the importance to ensure a higher level of clinical monitoring for patients with high baseline levels of CyC.

\section{LIMITATIONS}

This study has some limitations. First, this prospective observational study was conducted at a single center with a consequently limited sample size, thus the prognostic value of baseline CyC should be further confirmed in larger multicentric studies. Second, despite 
baseline data of our cohort were largely available and detailed for most of patients enrolled, the effect of some unknown and unmeasured confounders could have conditioned values of CyC (i.e. previous studies showed that the analysis of cystatin C is affected by hyperthyroidism, inflammation, rheumatoid factor and hypertriglyceridemia) (11). Data about procedural features (except from the volume of contrast media) and their impact on outcomes were not systematically collected as this was beyond the purpose of the present study. Despite CIAKI prophylaxis was strictly uniform as per department protocol, patients requiring urgent catheterization (i.e. admitted with STEMI diagnosis) did not receive a standard scheme of hydration to prevent such complication.

It could be argued that patients with highest levels of CyC were the ones with a previously known renal impairment (i.e. all had eGFR $<60 \mathrm{~mL} / \mathrm{min} / 1.73 \mathrm{~m} 2$ according to CKD-EPI equation) and consequently more exposed to CIAKI. However, the main purpose of this research was to show CyC accuracy in predicting or ruling out CIAKI even compared to standard and recognized markers of renal functionality, more than identifying novel markers of CIAKI. Moreover, the independent prognostic value of baseline CyC was confirmed by multivariate analysis for a major endpoint as cardiovascular death.

\section{CONCLUSIONS}

A baseline determination of $\mathrm{CyC}$ before $\mathrm{CA}$ is a powerful tool to rule out subsequent $\mathrm{CIAKI}$ and long-term adverse events. According to CyC baseline values, the clinician may be encouraged to perform additional CIAKI prophylaxis and a close follow-up in higher-risk patients as long as early discharge in patients at lower risk, with possible economic and practical advantages.

Acknowledgements

None. 


\section{Funding}

This research received no grant from any funding agency in the public, commercial or notfor-profit sectors.

\section{Disclosures}

The authors declare that there is no conflict of interest. 


\section{REFERENCES}

1. Anavekar, N. S. et al. Relation between Renal Dysfunction and Cardiovascular Outcomes after Myocardial Infarction. N. Engl. J. Med. 351, 1285-1295 (2004).

2. Coresh, J., Astor, B. C., Greene, T., Eknoyan, G. \& Levey, A. S. Prevalence of chronic kidney disease and decreased kidney function in the adult US population: Third National Health and Nutrition Examination Survey. Am. J. Kidney Dis. Off. J. Natl. Kidney Found. 41, 1-12 (2003).

3. Sadeghi, H. M. et al. Impact of Renal Insufficiency in Patients Undergoing Primary Angioplasty for Acute Myocardial Infarction. Circulation 108, 2769-2775 (2003).

4. Charytan, D. M., Li, S., Liu, J. \& Herzog, C. A. Risks of death and end-stage renal disease after surgical compared with percutaneous coronary revascularization in elderly patients with chronic kidney disease. Circulation 126, S164-169 (2012).

5. Gruberg, L. et al. The prognostic implications of further renal function deterioration within $48 \mathrm{~h}$ of interventional coronary procedures in patients with pre-existent chronic renal insufficiency. J. Am. Coll. Cardiol. 36, 1542-1548 (2000).

6. Kidney Disease: Improving Global Outcomes (KDIGO) Acute Kidney Injury Work Group. KDIGO Clinical Practice Guideline for Acute Kidney Injury. Kidney Int Suppl 2, 1-138 (2012).

7. Nash, K., Hafeez, A. \& Hou, S. Hospital-acquired renal insufficiency. Am. J. Kidney Dis. 39, 930-936 (2002).

8. Hou, S. H., Bushinsky, D. A., Wish, J. B., Cohen, J. J. \& Harrington, J. T. Hospitalacquired renal insufficiency: A prospective study. Am. J. Med. 74, 243-248 (1983).

9. Chertow, G. M., Burdick, E., Honour, M., Bonventre, J. V. \& Bates, D. W. Acute Kidney Injury, Mortality, Length of Stay, and Costs in Hospitalized Patients. J. Am. Soc. Nephrol. 16, 3365-3370 (2005). 
10. Solomon, R. \& Dauerman, H. L. Contrast-Induced Acute Kidney Injury. Circulation 122, 2451-2455 (2010).

11. Séronie-Vivien, S. et al. Cystatin C: current position and future prospects. Clin. Chem. Lab. Med. 46, (2008).

12. Herget-Rosenthal, S. et al. Early detection of acute renal failure by serum cystatin C. Kidney Int. 66, 1115-1122 (2004).

13. Briguori, C. et al. Cystatin C and Contrast-Induced Acute Kidney Injury. Circulation 121, 2117-2122 (2010).

14. Peng, L. et al. [Diagnostic value of cystatin C in contrast-induced acute kidney injury after percutaneous coronary intervention]. Zhonghua Nei Ke Za Zhi 54, 188-192 (2015).

15. Ribichini, F. et al. Comparison of Serum Creatinine and Cystatin C for Early Diagnosis of Contrast-Induced Nephropathy after Coronary Angiography and Interventions. Clin. Chem. 58, 458-464 (2012).

16. Liu, X. et al. Plasma neutrophil-gelatinase-associated lipocalin and cystatin C could early diagnose contrast-induced acute kidney injury in patients with renal insufficiency undergoing an elective percutaneous coronary intervention. Chin. Med. J. (Engl.) 125, 1051-1056 (2012).

17. Levey, A. S. et al. A new equation to estimate glomerular filtration rate. Ann. Intern. Med. 150, 604-612 (2009).

18. Usmiani, T. et al. AKIGUARD (Acute Kidney Injury GUARding Device) trial: in-hospital and one-year outcomes. J. Cardiovasc. Med. 1 (2015). J Cardiovasc Med (Hagerstown). 2017 Nov;18(11):908-915

19. Andreis A, Budano C, Levis M, Garrone P, Usmiani T, D'Ascenzo F, De Filippo O, D'Amico M, Bergamasco L, Biancone L, Marra S, Colombo A, Gaita F: Contrast-induced kidney injury: how does it affect long-term cardiac mortality? J Cardiovasc Med (Hagerstown). 2017 Nov;18(11):908-915 
20. Liu Y, Chen KH, Chen SQ, Chen LL, Duan CY, Wang K, Guo XS, Li HL, Bei WJ, Lin KY, Chen PY, Xian Y, Tan N, Zhou YL, Geng QS, Chen JY: "Predictive value of postprocedural early (within $24 \mathrm{~h}$ ) increase in cystatin C for contrast-induced acute kidney injury and mortality following coronary angiography or intervention. Oncotarget. 2017 Jul;8(65):109762-109771

21. Ishibashi Y, Yamauchi M, Musha H, Mikami T, Kawasaki K, Miyake F.Impact of contrastinduced nephropathy and cardiovascular events by serum cystatin C in renal insufficiency patients undergoing cardiac catheterization. Angiology. 2010 Nov;61(8):724-30

22. Tanaga K, Tarao K, Nakamura Y, Inoue T, Jo K, Ishikawa T, Miyazaki A. "Percutaneous coronary intervention causes increase of serum cystatin $C$ concentration even in the patients with a low risk of contrast-induced nephropathy." Cardiovasc Interv Ther. 2012 Sep;27(3):168-73

23. Padhy, M. et al. Serum neutrophil gelatinase associated lipocalin (NGAL) and cystatin C as early predictors of contrast-induced acute kidney injury in patients undergoing percutaneous coronary intervention. Clin. Chim. Acta. 435, 48-52 (2014).

24. Laders, F., Meyborg, M., Malyar, N. \& Reinecke, H. The Preinterventional CystatinCreatinine-Ratio: A Prognostic Marker for Contrast Medium-Induced Acute Kidney Injury and Long-Term All-Cause Mortality. Nephron 131, 59-65 (2015).

25. Yang, Y. et al. Comparison of Serum Cystatin $C$ and Creatinine Level Changes for Prognosis of Patients After Peripheral Arterial Angiography. Angiology 66, 766-773 (2015).

26. McCullough, P. A. Commentary: contrast-induced nephropathy and long-term adverse events: cause and effect? Nephrol. Dial. Transplant. 24, 3578-3579 (2009). 
27. Ichimoto E, Jo K, Kobayashi $\mathrm{Y}$, Inoue T, Nakamura $\mathrm{Y}$, Kuroda N, Miyazaki A, Komuro I.Prognostic significance of cystatin $C$ in patients with ST-elevation myocardial infarction. Circ J. 2009 Sep;73(9):1669-73. Epub 2009 Jul 13

28. Knight, E. L. et al. Factors influencing serum cystatin $\mathrm{C}$ levels other than renal function and the impact on renal function measurement. Kidney Int. 65, 1416-1421 (2004).

29. Deo, R. et al. Association of Cystatin C with Ischemia in Patients with Coronary Heart Disease. Clin. Cardiol. 32, E18-E22 (2009).

30. Patel, P. C. et al. Association of Cystatin C With Left Ventricular Structure and Function: The Dallas Heart Study. Circ. Heart Fail. 2, 98-104 (2009).

31. Moran A, Katz R, Smith NL, Fried LF, Sarnak MJ, Seliger SL, Psaty B, Siscovick DS, Gottdiener JS, Shlipak MG: Cystatin C concentration as a predictor of systolic and diastolic heart failure. J Card Fail. 2008 Feb;14(1):19-26

32. Luo J, Wang LP, Hu HF, Zhang L, Li YL, Ai LM, Mu HY, Kun-Wang: Cystatin C and cardiovascular or all-cause mortality risk in the general population: A meta-analysis. Clin Chim Acta. 2015 Oct 23;450:39-45

\section{FIGURE LEGENDS}

Figure 1

Panel a: ROC curve showing correlation between baseline CyC values and incidence of CIAKI Panel b: comparison between baseline $\mathrm{CyC}$ and other markers of renal functionality as predictors of CIAKI.

Abbreviations.

$A \cup C=$ area under the curve; $C y C=$ cystatin $C ; C K D E P I:$ Chronic Kidney Disease Epidemiology Collaboration (equation); eGFR= estimated glomerular filtration rate; MDRD= Modification of Diet in 
Renal Disease Study (equation); sCR= serum creatinine; Cockroft refers to Cockroft and Gault equation

Figure 2:

Kaplan-Meyer analysis showing survival rates (panel A), freedom from cardiovascular death (panel b) and from MACE (panel c) according to baseline value of Cystatin C.

Abbreviations.

CyC $=$ cystatin $C ; M A C E=$ major adverse cardiovascular events. 